\title{
NOTE ON FIELDS OF SMALL DISCRIMINANT
}

\section{HARVEY COHN ${ }^{1}$}

The problem of finding totally real fields of any degree and small discriminant is of interest, aside from intrinsic reasons, because of its connection with critical lattices for the product of the coordinates. The ground-work, however, seems to be largely unexplored. This is probably illustrated by a somewhat persistent conjecture [4] that for totally real fields of degree $(g-1) / 2$, where $g$ is a prime $>3$, the smallest discriminant is $g^{(0-3) / 2}$, achieved for $R(\cos 2 \pi / g)$, where $R$ is the field of rational numbers. (This is the maximal real sub-field in the field of the $g$ th roots of unity.) This conjecture was often made implicitly even before its verification [1] by Davenport for $g=7$ (the case $g=5$ having been trivial). We disprove this for those $g$, such as 13,37 , etc., for which $(g+1) / 2$ is also prime. We use relative quadratic fields built on base fields of small discriminant, a device used previously by Mayer [3] in his work on biquadratic fields.

The counterexamples are constructed by adjoining to the totally real base-field $k$ the quantity $\mu^{1 / 2}$, where $\mu$ is a totally positive integer in $k$ which is not a perfect square; we call $k\left(\mu^{1 / 2}\right)=K$. Next we consider the prime ideal $p$ dividing the odd rational prime $p$ and the prime ideal $q$ dividing 2 , in the ground-field $k$. Then according to the well known methods of Hilbert [2] the discriminant $D$, of $K$, and the discriminant $d$, of $k$, are related by $D=d^{2} N(\delta)$ where $N(\delta)$ is the norm (over $R$ ) of the relative discriminant $b$. Assuming for convenience that $d$ is odd, we have $\mathfrak{d}=\subseteq \mathfrak{T}^{2}$ where $\mathfrak{S}$ is the product of all $\mathfrak{p}$ and $\mathfrak{q}$ that divide $(\mu)$ to an odd power, and $\mathfrak{T}$ is the product of all $q$ for which the congruence $\mu \equiv \zeta^{2} \bmod q^{2+e}$ is unsolvable for $\zeta$ an integer in $k$, with $e$ equal to the exponent of the power to which $q$ divides $(\mu)$. In the cases worked out fully (below) it will prove advantageous to choose $\mu$ relatively prime to 2 and such that the congruence $\mu \equiv \zeta^{2}$ mod 4 is solvable, for then $\mathfrak{T}=(1)$.

We take for the base-field $k=R(\cos 2 \pi / l)$, of degree $(l-1) / 2$, where $l$ is a prime $>5$. For this field $d=l^{(l-3) / 2}$.

(i) First we take $\mu=5$. Since (5) is unramified and $\mu \equiv 1 \bmod 4$, then $\delta=(\mu)$ and $N(\delta)=5^{(l-1) / 2}$. Therefore $K^{(1)}=R\left(\cos 2 \pi / l, 5^{1 / 2}\right)$ defines a totally real field of degree $l-1$ with discriminant $D^{(1)}$ $=l^{l-3} 5^{(l-1) / 2}$. 1952.

Presented to the society, December 28,1951 ; received by the editors January 15 ,

1 Research currently sponsored by the U. S. Army O.O.R.

2 Numbers in brackets are items cited in the bibliography at the end. 
(ii) Secondly we take $\mu=3(2-2 \cos 2 \pi / l)$. Since (3) is unramified and $\mu \equiv(2 \cos \pi / l)^{2} \bmod 4$, then, once more, $\delta=(\mu)$, and $N(b)$ $=3^{(l-1) / 2} l$. Therefore $K^{(2)}=R\left((3(2-2 \cos 2 \pi / l))^{1 / 2}\right)$ is a totally real field of degree $l-1$ with discriminant $D^{(2)}=l^{l-2} 3^{(l-1) / 2}$.

Now whenever $2 l-1$ (called $g$ earlier) is a prime, as well as $l$, the totally real fields $K^{(1)}$ (when $l=7$ ) and $K^{(2)}$ (when $l=19,31,37, \cdots$ ) of degree $l-1$ provide smaller discriminants than the conjectured field, $R(\cos 2 \pi /(2 l-1))$, of discriminant $(2 l-1)^{l-2}$ and degree $l-1$. In fact when $l=7$ the field $K^{(1)}$ provides the smallest discriminant for a quadratic extension of the indicated base-field (although the details of this last step can be omitted as they fall within the earlier outline of Hilbert's methods).

\section{BIBLIOGRAPHY}

1. H. Davenport, On the product of three homogeneous linear forms. III, Proc. London Math. Soc. vol. 45 (1939) pp. 98-125.

2. D. Hilbert, Über die Theorie des relativquadratischen Zahlkörpers, Math. Ann. vol. 51, pp. 1-127.

3. J. Mayer, Die absolut-kleinsten Diskriminanten der biquadratischen Zahlkörper, Sitzungsberichte der Akademie der Wissenschaften Wien Abt. IIa vol. 138 (1929) pp. 733-742.

4. C. L. Siegel, Geometry of numbers, mimeographed lecture notes, New York University, 1946, Chap. IX, p. 14.

WAYNE UNIVERSITY 